\title{
Comparative Evaluation of Antifungal Activity of Octenidine: An In Vitro Confocal Laser Study
}

\author{
N Bharath Naga Reddy ${ }^{1}$, Dinesh Sridhar ${ }^{2}$, Arasappan Rajkumar ${ }^{3}$, Sabari Murugesan ${ }^{4}$, Karthikeyan Selvaraj ${ }^{5}$, Sathish Sankar ${ }^{6}$
}

\begin{abstract}
Aim: The aim of this study was to evaluate the antifungal efficacy of a novel endodontic irrigant octenidine against conventional irrigants sodium hypochlorite and EDTA on Candida albicans growth in the young and old population by calculating the number of colonies formed and by qualitative identification of dead/viable fungi by Confocal laser scanning microscopy (CLSM) method.

Materials and methods: The total number of samples used in the study was eighty samples after decoronation of the crown portion the middle third of root canal. Each group was subdivided into four subgroups with various irrigation protocols: (A) $17 \% \mathrm{EDTA}+5.25 \% \mathrm{NaOCl}$, (B) $100 \%$ Octenisept, (C) 17\% EDTA + 5.25\% NaOCl + 1\% clotrimazole, and (D) phosphate buffer saline. After completion of irrigation ATCC samples (90028) of C. albicans were inoculated with $5 \mathrm{~mL}$ of peptone water each and incubated at $37^{\circ} \mathrm{C}$ for 72 hours to attain the turbidity corresponding to 0.5 McFarland standards CFU. Eight samples were analyzed for the formation of candidal colonies, and two samples for the assessment of viability of Candida by confocal laser scanning microscope in each subgroup.

Results: Comparison of antifungal efficacy of endodontic irrigants employed in the young and old populations revealed a significant reduction in the mean values of CFU and the mean values of percentage of non-viable microorganism by CLSM method. A positive relationship was revealed in the younger population which had a better antifungal efficacy than the older population in all the irrigant subgroups evaluated in the study. Conclusion: All the endodontic irrigants employed in our study had a good antifungal efficacy against Candida albicans. Octenisept had a maximum antifungal efficacy, while phosphate saline showed the least efficacy in both age groups, which was quantitatively evaluated by CFU method, and the same was confirmed through qualitative evaluation by CLSM method.

Clinical significance: Candida albicans plays a major role in the establishment and pathogenesis of failed root canal treatment. Age-related changes alter the adhesion potential of dentin, in turn influence the outcome of endodontic therapy. Octenidine, a novel antifungal agent, can be substituted over the conventionally used EDTA and $\mathrm{NaOCl}$ with less adverse effects.

Keywords: Antifungal activity, Candida albicans, Confocal laser scanning microscope, Endodontic irrigants.

The Journal of Contemporary Dental Practice (2020): 10.5005/jp-journals-10024-2860
\end{abstract}

\section{INTRODUCTION}

Microbial agents play a primary role in the establishment of pulp and periradicular diseases. ${ }^{1}$ The incidence of endodontic failure possesses an enigma to the endodontist, predominantly due to the diversity of the microbial species involved in the secondary infections. ${ }^{2}$ The mechanical instrumentation helps to debulk the microbial load and to combat the microbial challenge created by the residual microbes in the failed root canal cases, which has tended us to use antifungal agents as endodontic irrigants. ${ }^{3}$ Candida albicans is a dentinophilic yeast which is reported in 10-14 percentages of secondary infections. The unique features are its adaptive capacity toward survival in the absence of nutrients and in wide range of $\mathrm{pH}^{4}$ This along with penetration into the dentinal tubule and capacity to form hyphae and blastospores helps in the progression of the infection. ${ }^{5}$

Sodium hypochlorite has been predominantly used because of its antimicrobial, sporicidal, fungicidal, and tissue-dissolving properties which act on the organic components of the dentin substrate. ${ }^{6}$ Its usage has to be confined within the root canal system and can cause hypochlorite accident if it is used without adequate precautions. ${ }^{7}$ EDTA was first introduced to the endodontic literature by Nygaard-Ostby. The mechanism of action is predominantly on the inorganic component of dentin, and it reacts with calcium ions in dentin and forms soluble calcium chelate, which reduces the availability of calcium ions essential for the pathogenesis of candidal infections. ${ }^{8}$
1,5,6Department of Conservative Dentistry and Endodontics,
Adhiparasakthi Dental College and Hospital, Tamil Nadu Dr. MGR
Medical University, Melmaruvathur, Tamil Nadu, India
${ }^{2}$ Department of Conservative Dentistry and Endodontics, Sri
Venkateshwara Dental College, Pondicherry University, Puducherry,
India
${ }^{3}$ Department of Conservative Dentistry and Endodontics, Sri
Ramachandra Institute of Higher Education and Research, Chennai,
Tamil Nadu, India
${ }^{4}$ Department of Restorative Dental Sciences, College of Dentistry,
Jazan University, Jazan, Kingdom of Saudi Arabia
Corresponding Author: N Bharath Naga Reddy, Department of
Conservative Dentistry and Endodontics, Adhiparasakthi Dental
College and Hospital, Melmaruvathur, Tamil Nadu Dr. MGR Medical
University, Tamil Nadu, India, Phone: +91 9444792260, e-mail:
drbharathendo999@gmail.com

How to cite this article: Bharath Naga Reddy N, Sridhar D, Rajkumar A, et al. Comparative Evaluation of Antifungal Activity of Octenidine: An In Vitro Confocal Laser Study. J Contemp Dent Pract 2020;21(8): 905-909.

Source of support: Nil

Conflict of interest: None

Clotrimazole is an antifungal agent with a broad spectrum of action. Chandra et al. has used clotrimazole as an additional

() The Author(s). 2020 Open Access This article is distributed under the terms of the Creative Commons Attribution 4.0 International License (https://creativecommons. org/licenses/by-nc/4.0/), which permits unrestricted use, distribution, and non-commercial reproduction in any medium, provided you give appropriate credit to the original author(s) and the source, provide a link to the Creative Commons license, and indicate if changes were made. The Creative Commons Public Domain Dedication waiver (http://creativecommons.org/publicdomain/zero/1.0/) applies to the data made available in this article, unless otherwise stated. 
antifungal agent and found that it exerts a synergistic action along with routine irrigation protocols employed for disinfection against Candida. ${ }^{9}$ Octenisept has been employed as an antiseptic for skin burns and wound disinfection. Octenidine hydrochloride has been suggested as a potential endodontic irrigant based on its antimicrobial effects and low cytotoxicity. It has a broad spectrum of activity against fungi and viruses, which has not been explored. This has tended us to select it as the primary endodontic irrigant. ${ }^{10}$

Age-related changes alter the adhesion capability of dentin which influences the outcome of endodontic therapy. Candida albicans has preponderance to get attached to the radicular dentin and exerts a collagenolytic action which affects the prognosis of treatment. $^{11}$

The usage of the novel irrigant octenidine in the extreme age groups has not been evaluated in the literature previously.

We in this study evaluated the antifungal efficacy of the novel endodontic irrigant octenidine against conventional irrigants sodium hypochlorite and EDTA on Candida albicans in the young and old populations by calculating the number of colonies formed and by qualitative identification of dead/viable fungi by CLSM method.

\section{Materials and Methods}

The ethical clearance for the study was obtained from the IRB and ethics committee of APDCH. The total number of tooth samples included in the study was 80 , of which forty tooth samples were collected from young population aged between 12 years and 25 years, which were extracted for orthodontic reasons, and the remaining forty tooth specimens [40] were collected from older population aged above 60 years, which were extracted for periodontal and prosthodontic reasons. The samples were obtained from the Oral Surgery Department, Adhiparasakthi Dental College and Hospital, Melmaruvathur.

\section{Sample Preparation}

The teeth were cleaned and subjected to thermocycling, which were then stored in artificial saliva procured from laboratory for a week to simulate the oral environment. Decoronation was done, and the remaining portion was subjected to pulp extirpation with broaches. A $25-\mathrm{mm}$, size $15 \mathrm{~K}$-File was used to determine the working length, and the orifice was enlarged with Gates Glidden drill size 2 and the apical preparation was done until ISO size 50. The irrigants employed in the study were subdivided into four subgroups with10 samples for each subgroup, and the following irrigation protocols were employed: [A] $2 \mathrm{~mL}$ of $17 \%$ EDTA for one min and $2 \mathrm{~mL}$ of $5.25 \% \mathrm{NaOCl}$ for one min, [B] $2 \mathrm{~mL}$ of Octenisept for one $\mathrm{min}$, and [C] $2 \mathrm{~mL}$ of $17 \%$ EDTA for one min and $2 \mathrm{~mL}$ of $5.25 \%$ $\mathrm{NaOCl}$ for one min. The middle third of the root canal was sectioned and employed for the study. Microbial aspects of the study were done at the Department of Microbiology, MAPIMS, Melmaruvathur.

The ingredients of the prepared Sabouraud dextrose broth include [1] special peptone $10 \mathrm{~g} / \mathrm{L}$, and [2] dextrose $40 \mathrm{~g} / \mathrm{L}$, the $\mathrm{pH}$ of which is adjusted to 5.6. A $6.5 \mathrm{~g}$ agar was weighed and dissolved in $100 \mathrm{~mL}$ of distilled water in a conical flask. The conical flask was plugged with cotton and sterilized in autoclave at $121^{\circ} \mathrm{C}$ for 15 minutes at $15 \mathrm{lbs}$ pressure. The sterilized Sabouraud dextrose agar was poured into petri dishes to a depth of $5 \mathrm{~mm}$ under the laminar flow chamber. A quantity of $100 \mathrm{~mL}$ of medium was used to pour six culture plates. The poured plates were allowed to solidify and were refrigerated.

\section{Inoculation of Samples}

Dentin shavings were collected with Gates Glidden burs [0.9 $\mathrm{mm}]$, and the dislodgement of samples was done using sonics. The dentin shavings collected were inoculated with $0.3 \mathrm{~mL}$ of the adjusted C. albicans suspension. Candida albicans (ATCC 90028) was the test organism chosen in this investigative study and was procured from Dr. Lal Microbiological Culture Lab in Chennai. The inoculums with dentin scrapings were kept in the incubator for 72 hours. The replenishment of samples was done at regular periodicity of one day with fresh candidal suspension broth. After a two-day period, the aliquots were taken using a syringe and transferred using a one-micron inoculation loop on a $4 \%$ Sabouraud dextrose agar plate for verification of Candida albicans growth, and the mean and standard deviation were calculated for the number of colony-forming units.

\section{Dyes and Kits}

Samples preparation was done using the hard tissue microtome, SYTO9, and propidium iodide (PI) staining agents employed for CLSM evaluation.

\section{Colony-forming Unit Evaluation}

Among the ten dentin disks in every subgroup eight disks were quantitatively assessed for formation of colonies (CFU).

\section{Confocal Laser Evaluation}

Each cross section was examined under a confocal laser scanning microscope and was recorded at $63 \times$ magnification employing fluorescent mode with a laser illumination source. Two samples in each subgroup were assessed qualitatively to detect the viability, that is the presence of live or dead fungi in the dentinal tubules.

\section{Results}

Comparison of the antifungal efficacy of the endodontic irrigants employed in the young and old populations revealed a significant reduction in the mean values of CFU. A positive relationship was revealed between the mean values of CFU, while more reduction was revealed in the younger population than in the older population in all irrigant subgroups evaluated in the study.

In this study one-way ANOVA followed by Tukey's HSD test showed statistically significant difference among various subgroups concerning the discrepancy in the colony-forming units in each group. Unpaired $t$-test showed a significant difference in the number of colony-forming units between the groups.

\section{Comparison of Antifungal Efficacy by CFU Method}

Octenidine subgroup had a highest reduction in CFU with mean values of 9.38 and 38.75 in the young and old populations, respectively, and was the most efficient one (Fig. 1 and Table 1). Among the other irrigants evaluated phosphate saline subgroup had the least reduction in CFU with mean values of 570.63 and 997.25 in the young and old populations, respectively, and hence was the least efficient one (Fig. 2 and Table 1).

Clotrimazole subgroup had a significantly better reduction with mean values of 14.88 and 51.13 in the young and old populations, respectively (Table 1), compared to sodium hypochlorite/EDTA subgroup with mean values of 62.25 and 185.75 in the young and old populations, respectively [(Table 1$)$. There was a positive but nonsignificant relation between octenidine and clotrimazole 


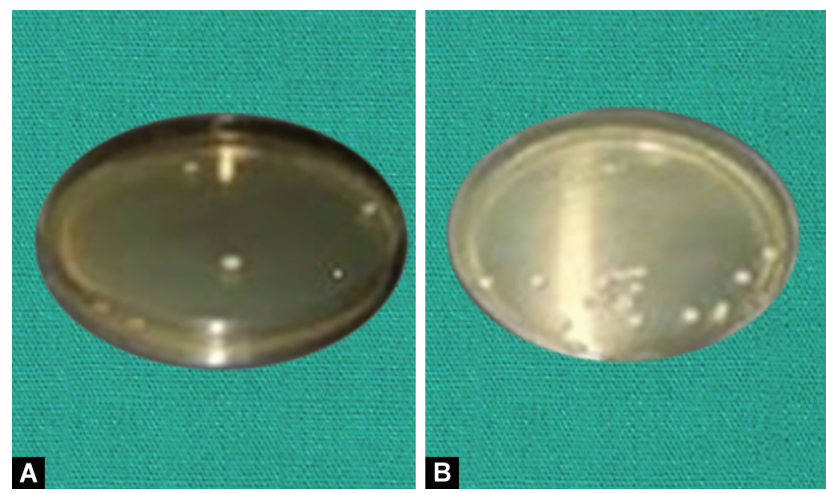

Figs $1 \mathrm{~A}$ and $\mathrm{B}$ : CFU image of octenidine group

Table 1: Comparison of CFU between the groups

\begin{tabular}{lll}
\hline Groups & Young-I (mean \pm SD) & Old-II (mean \pm SD) \\
\hline $\begin{array}{l}\text { I } 17 \% \text { EDTA }+5.25 \% \\
\text { NaOCI }\end{array}$ & $62.25 \pm 8.73$ & $185.75 \pm 16.36$ \\
II Octenisept & $9.38 \pm 3.02$ & $38.75 \pm 6.23$ \\
III $17 \% \mathrm{EDTA}+$ & $14.88 \pm 4.49$ & $51.13 \pm 10.87$ \\
$\begin{array}{l}5.25 \% \mathrm{NaOCl}+1 \% \\
\text { Clotrimazole }\end{array}$ & \\
$\begin{array}{l}\text { IV Phosphate buffer } \\
\text { saline }\end{array}$ & $570.63 \pm 33.02$ & $997.25 \pm 74.66$ \\
$p$ value & & \\
\hline
\end{tabular}

subgroups with $p$ values of 0.919 and 0.918 in the young and old groups, respectively, suggesting both irrigant combinations were equally effective and are represented in Table 2.

\section{Confocal Laser Results Analysis}

The viability of microbes read as red and green voxels for dead and live organisms, respectively, and the mean values of percentage of non-viable microorganism were calculated employing special software.

Octenidine subgroup had the highest reduction with the presence of $17 \%$ and $15 \%$ of non-viable microorganism in the young and old populations, respectively, and was the most efficient one (Fig. 3 and Table 3)]. Among the other irrigants evaluated phosphate saline subgroup had the least reduction with the presence of $7 \%$ and $5 \%$ of non-viable microorganism in the young and old populations, respectively, and was the least efficient one (Fig. 4 and Table 3).

Clotrimazole subgroup had a significantly better reduction with the presence of $12 \%$ and $10 \%$ of non-viable microorganism in the young and old populations, respectively (Table 3), compared to sodium hypochlorite/EDTA subgroup with the presence of $8 \%$ and $6 \%$ of non-viable microorganism in the young and old populations, respectively (Table 3 ).

There was a positive but nonsignificant relation between octenidine and clotrimazole subgroups with $p$ values of 0.719 and 0.710 in the young and old groups, respectively, suggesting both irrigant combinations were equally effective as represented in (Table 4).

\section{Discussion}

Residual microbes with diverse endodontic flora lead to compromised outcome of root canal therapy and require

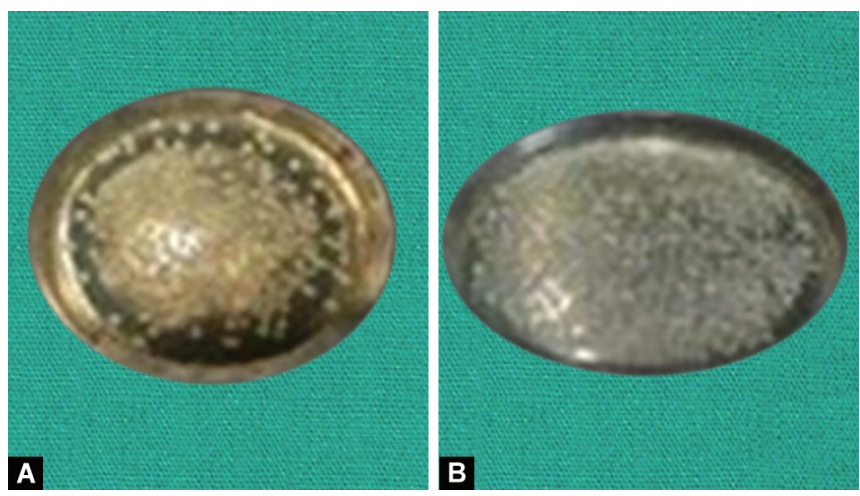

Figs $2 \mathrm{~A}$ and $\mathrm{B}$ : CFU image of phosphate saline group

Table 2: Significance of fungal growth among the four irrigants

\begin{tabular}{lll}
\hline Groups & Young-I ( $p$ value) & Old-II ( $p$ value $)$ \\
\hline I vs II & 0.000 & 0.000 \\
I vs III & 0.000 & 0.000 \\
I vs IV & 0.000 & 0.000 \\
II vs III & 0.919 & 0.917 \\
II vs IV & 0.000 & 0.000 \\
III vs IV & 0.000 & 0.000 \\
\hline
\end{tabular}

employment of novel strategies in management. The unique features of the above study were to compare the antifungal potency of the newer endodontic irrigant Octenisept against Candida organism with exploration of its significance in different age groups. This study also employed a novel evaluation method to directly estimate the viability of organism.

This comparative study revealed a significant variation among the mean values of CFU and the percentage of nonviable microorganism remaining after the irrigation protocols of all the four tested irrigants. There was no statistically significant difference between octenidine and clotrimazole in both age groups, suggesting both irrigant combinations were equally effective. Clotrimazole greatly augmented the antifungal effect against Candida albicans in both age groups as it interferes with the synthesis of ergosterol causing disruption of the cell membrane. Baumgartner et al. revealed E. faecalis and Candida albicans as the most common organisms associated with root canal failure. ${ }^{12}$

Octenidine subgroup had the highest reduction in CFU with mean values of 9.38 in young population and 38.75 in old population. A higher amount of calcium present in the old age group contributes to the stabilization of bond established between the Candida albicans and dentin. ${ }^{13}$ The dimension of fungi was found to be smaller than the size of obliterated dentinal tubules associated with aging. The surface molecules with invasive affinity mediates the adherence of Candida to the collagen component of dentin. ${ }^{14}$

The sodium hypochlorite/EDTA subgroup with mean values of 62.25 and 185.75 in the young and old age groups, respectively, ranks third with the presence of $8 \%$ of non-viable microorganism in the young age group and 6\% in the older age group. The high $\mathrm{pH}$ of sodium hypochlorite along with hydroxyl ions generation interferes with the integrity of cytoplasmic membrane causing irreversible damage to the cell wall. ${ }^{15}$ Ates et al. revealed that $17 \%$ EDTA had anti-colonization and anti-collagenolytic properties against $C$. albicans, which prevents its binding and reduces the 

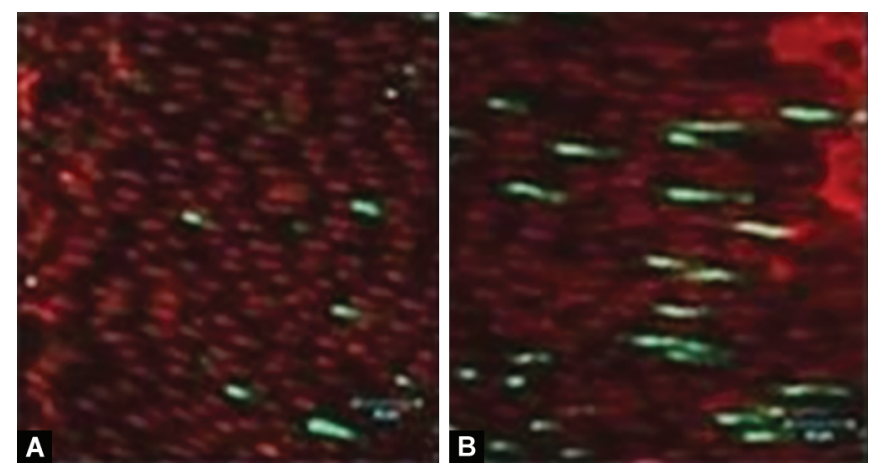

Figs $3 \mathrm{~A}$ and $\mathrm{B}$ : CLSM image of octenidine group at $63 \times$ magnification

Table 3: Yeast viability of the two groups

\begin{tabular}{lll}
\hline Groups & $\begin{array}{l}\text { Young-I (mean } \pm \\
\text { SD) (\%) }\end{array}$ & $\begin{array}{l}\text { Old-II (mean } \pm \text { SD) } \\
(\%)\end{array}$ \\
\hline I 17\% EDTA + 5.25\% & 8 & 6 \\
NaOCI & & \\
II Octenisept & 17 & 15 \\
III 17\% EDTA + & 12 & 10 \\
$5.25 \% \mathrm{NaOCI}+1 \%$ & & \\
Clotrimazole & & 5 \\
IV Phosphate buffer & 7 & 5 \\
saline & & 0.000 \\
$p$ value & 0.000 & \\
\hline
\end{tabular}

growth by removing calcium through chelation from the cell walls and aids in collapse of the cell wall. ${ }^{16}$

Techniques available for the quantitative evaluation of bacterial flora includes histologic sections, scanning electron microscope, and microbiological assays. Microbiological sampling techniques estimate the number of colony-forming units they do read on spatial distribution of bacteria in tubules. The histologic sections show the distribution of the bacteria in the infected dentin, but lack the details of viability.

In consideration of the above facts confocal laser scanning microscopy (CLSM) becomes important because it is a suitable method for the identification of live and dead bacteria inside the tubules to analyze the viability profile, architecture, and spatial distribution. We in this study employed the CLSM for the qualitative evaluation of bacterial flora, and according to Vitkov et al., SYTO9 probe [473 nm] was used for the evaluation of live bacteria and propidium iodide probe [ $559 \mathrm{~nm}]$ for the evaluation of dead bacteria as it penetrates the damaged plasma membrane. The contact time for dye was kept as 5 minutes at 1:1 ratio.

The stains were assessed for fluorescence, and the images were captured by special software at a resolution of 512 pixels 2 and with a zoom factor of 1.0, giving a final pixel resolution of 0.41 $\mathrm{mm} /$ pixel. ${ }^{17}$ The antifungal efficacy of octenidine might be related to the fact that it resists the challenges created by organic and inorganic inhibitory factors in the root canal system. ${ }^{17}$ It maintains its antimicrobial efficacy in the presence of organic material in the biologically complex environment and exerts a substantive effect on dentin. ${ }^{18}$ This was in accordance with Tirali et al. who demonstrated that $100 \%$ Octenisept was more effective than $5.25 \%$ $\mathrm{NaOCl}$ as an antimicrobial endodontic irrigant. ${ }^{19-22}$

Tandjung et al. investigated the biocompatibility and safety aspects of Octenisept on E. faecalis and concluded that toxicity
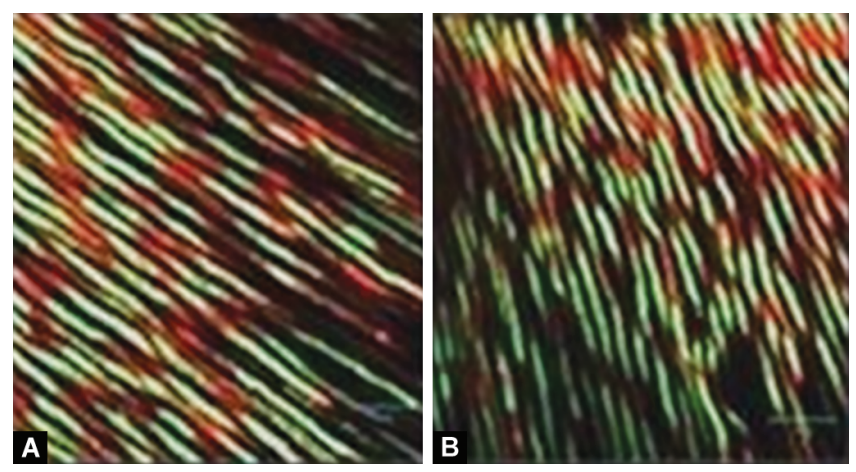

Figs 4A and B: CLSM image of phosphate saline group at $63 \times$ magnification

Table 4: Significance of yeast viability among the four irrigants

\begin{tabular}{lll}
\hline Groups & Young-I ( $p$ value) & Old-II ( $p$ value $)$ \\
\hline I vs II & 0.000 & 0.000 \\
I vs III & 0.000 & 0.000 \\
I vs IV & 0.000 & 0.000 \\
II vs III & 0.719 & 0.710 \\
II vs IV & 0.000 & 0.000 \\
III vs IV & 0.000 & 0.000 \\
\hline
\end{tabular}

parameters were all within the compliant limits. No carcinogenic or mutagenic effects have been reported, which has tended us to select it for our study. ${ }^{23}$ Results obtained in the CFU method were reflected in the CLSM evaluation with the presence of more C. albicans in the older population as compared to the young population.

\section{LIMITATIONS OF THE STUDY}

The effectiveness of endodontic irrigants needs to be evaluated by clinical trials with protocols to assess the effect of inflammatory mediators on the establishment and progression of secondary infections. Furthermore, there is no direct method to assess the antifungal effect and we rely on the indirect methods to assess the clinical scenario.

\section{Conclusion}

Within the limitations of our study it can be concluded that all the endodontic irrigants employed in our study had a good antifungal efficacy against Candida albicans.

Octenisept had a maximum antifungal efficacy, while phosphate saline showed the least efficacy in both age groups, which was quantitatively evaluated by the CFU method, and the same was confirmed through the qualitative evaluation by CLSM method.

In future, studies need to be concentrated to find out the antimicrobial effectiveness in clinical trials as it has better validity and reliability.

\section{References}

1. Kakehashi S, Stanley HR, Fitzgerald RJ. The effects of surgical exposures of dental pulps in germ-free and conventional laboratory rats. Oral Surgery, Oral Medicine, Oral Pathology 1965;20(3):340-349. DOI: 10.1016/0030-4220(65)90166-0.

2. Waltimo TM, Orstavik $D$, Sirén EK, et al. In vitro yeast infection of human dentin. J Endod 2000;26(4):207-209. DOI: 10.1097/00004770200004000-00002. 
3. Siqueira $J F$, Rocas $I N$, Lopes $H P$, et al. Fungal infection of the radicular dentin. J Endod 2002;28(11):770-773. DOI: 10.1097/00004770200211000-00006.

4. Brändle N, Zehnder M, Weiger R, et al. Impact of growth conditions on susceptibility of five microbial species to alkaline stress. J Endod 2008;34(5):579-582. DOI: 10.1016/j.joen.2008.02.027.

5. Şen BH, Safavi KE, Spangberg LS. Growth patterns of Candida albicans in relation to radicular dentin. Oral Surg Oral Med Oral Pathol Oral Radiol Endod 1997;84(1):68-73.

6. Şen BH, Safavi KE, Spangberg LS. Antifungal effects of sodium hypochlorite and chlorhexidine in root canals. J Endod 1999;25(4): 235-238. DOI: 10.1016/S0099-2399(99)80149-6.

7. Sena NT, Gomes BPFA, Vianna ME, et al. In vitro antimicrobial activity of sodium hypochlorite and chlorhexidine against selected singlespecies bio films. Int Endod J 2006;39(11):878-885. DOI: 10.1111/j.13652591.2006.01161.x.

8. Ozdemir HO, Buzoglu HD, Calt S, et al. Effect of ethylenediaminetetraacetic acid and sodium hypochlorite irrigation on Enterococcus faecalis biofilm colonization in young and old human root canal dentin: in vitro study. J Endod 2010;36(5):842-846. DOI: 10.1016/j. joen.2010.01.008.

9. Chandra SS, Miglani R, Srinivasan MR, et al. Antifungal efficacy of $5.25 \%$ sodium hypochlorite, $2 \%$ chlorhexidine gluconate, and $17 \%$ EDTA with and without an antifungal agent. J Endod 2010;36(4): 675-678. DOI: 10.1016/j.joen.2010.01.015.

10. Tandjung L, Waltimo T, Hauser I, et al. Octenidine in root canal and dentine disinfection ex vivo. Int Endod J 2007;40(11):845-851. DOI: 10.1111/j.1365-2591.2007.01279.x.

11. Kakoli $P$, Nandakumar R, Romberg $E$, et al. The effect of age on bacterial penetration of radicular dentin. J Endod 2009;35(1):78-81. DOI: 10.1016/j.joen.2008.10.004.

12. Baumgartner JC, Watts CM, Xia T. Occurrence of Candida albicans in infections of endodontic origin. J Endod 2000;26(12):695-698. DOI: 10.1097/00004770-200012000-00003.

13. Venegas SC, Palacios JM, Apella MC, et al. Calcium modulates interactions between bacteria and hydroxyapatite. J Dent Res 2006;85(12):1124-1128. DOI: 10.1177/154405910608501211.

14. Kinney JH, Nalla RK, Pople JA, et al. Age-related transparent root dentin: mineral concentration, crystallite size, and mechanical properties. Biomaterials 2005;26(16):3363-3376. DOI: 10.1016/ j.biomaterials.2004.09.004.

15. Radcliffe $C E$, Potouridou $L$, Qureshi R, et al. Antimicrobial activity of varying concentrations of sodium hypochlorite on the endodontic microorganisms actinomyces Israeli, A. Naes/undii, Candida albicans and Enterococcus faecalis. Int Endod J 2004;37(7):438-446. DOI: 10.1111/j.1365-2591.2004.00752.x.

16. Ates $M$, Akdeniz BG, Sen BH. The effect of calcium chelating or binding agents on Candida albicans. Oral Surg Oral Med Oral Pathol Oral Radiol Endod 2005;100(5):626-630. DOI: 10.1016/ j.tripleo.2005.03.004.

17. Jin $\mathrm{Y}$, Zhang $\mathrm{T}$, Samaranayake $\mathrm{YH}$, et al. The use of new probes and stains for improved assessment of cell viability and extracellular polymeric substances in Candida albicans biofilm. Mycopathologia 2005;159(3):353-360. DOI: 10.1007/s11046-004-6987-7.

18. Fidalgo TK, Barcelos R, Portela MB, et al. Inhibitory activity of root canal irrigants against Candida albicans, Enterococcus faecalis and Staphylococcus aureus. Braz Oral Res 2010;24(4):406-412. DOI: 10.1590/ s1806-83242010000400006.

19. Tirali RE, Turan $\mathrm{Y}$, Akal N, et al. In vitro antimicrobial activity of several concentrations of $\mathrm{NaOCl}$ and octenisept in elimination of endodontic pathogens. Oral Surg Oral Med Oral Pathol Oral Radiol Endod 2009;108(5):e117-e120. DOI: 10.1016/j.tripleo.2009. 07.012.

20. Turk BT, Ates $M$, Sen BH. The effect of treatment of radicular dentin on colonization patterns of C. Albicans. Oral Surg Oral Med Oral Pathol Oral Radiol Endod 2008;106(3):457-462. DOI: 10.1016/ j.tripleo.2008.05.012.

21. Sen BH, Chugal NM, Liu $H$, et al. A new method for studying the adhesion of Candida albicans to dentin in the presence or absence of smear layer. Oral Surg Oral Med Oral Pathol Oral Radiol Endod 2003;96(2):201-206. DOI: 10.1016/s1079-2104(03)00165-3.

22. Douglas LJ. Candida biofilms and their role in infection. Trends Microbiol 2003;11(1):30-36. DOI: 10.1016/s0966-842x(02) 00002-1.

23. Vianna ME, Gomes BPFA, Berber VB, et al. In vitro evaluation of the antimicrobial activity of chlorhexidine and sodium hypochlorite. Oral Surg Oral Med Oral Pathol Oral Radiol Endod 2004;97(1):79-84. DOI: 10.1016/s1079-2104(03)00360-3. 